\title{
SUPERCONVERGENCE ANALYSIS OF THE POLYNOMIAL PRESERVING RECOVERY FOR ELLIPTIC PROBLEMS WITH ROBIN BOUNDARY CONDITIONS*
}

\author{
$\mathrm{Yu} \mathrm{Du}$ and Haijun $\mathrm{Wu}$ \\ Department of Mathematics, Xiangtan University, Xiangtan 411105, China \\ Email: duyu@xtu.edu.cn, hjw@nju.edu.cn \\ Zhimin Zhang \\ Beijing Computational Science Research Center, Beijing 100193, China \\ Department of Mathematics, Wayne State University, Detroit, MI 48202, USA \\ Email: zmzhang@csrc.ac.cn, ag7761@wayne.edu
}

\begin{abstract}
We analyze the superconvergence property of the linear finite element method based on the polynomial preserving recovery (PPR) for Robin boundary elliptic problems on triangulartions. First, we improve the convergence rate between the finite element solution and the linear interpolation under the $H^{1}$-norm by introducing a class of meshes satisfying the Condition $(\alpha, \sigma, \mu)$. Then we prove the superconvergence of the recovered gradients post-processed by PPR and define an asymptotically exact a posteriori error estimator. Finally, numerical tests are provided to verify the theoretical findings.
\end{abstract}

Mathematics subject classification: 65N12, 65N15, 65N30.

Key words: Superconvergence, Polynomial preserving recovery, Finite element methods, Robin boundary condition.

\section{Introduction}

Let $\Omega \subset \mathbb{R}^{2}$ be a bounded polygon with boundary $\Gamma:=\partial \Omega$. Let $\mathbf{n}$ be the unit normal vector to the boundary exterior to $\Omega$. We consider the supercenvergence analysis for the model problem: Find $u \in H^{1}(\Omega)$ such that

$$
a(u, v):=\int_{\Omega}(\nabla u \cdot \nabla v+c u v)+\int_{\partial \Omega} q u v=f(v)+g(v), \quad \forall v \in H^{1}(\Omega),
$$

where $c \in L^{\infty}, q \in L^{\infty}(\Gamma), f \in H^{-1}(\Omega)$ and $g \in H^{-\frac{1}{2}}(\partial \Omega)$. We note that most results hold for a general class of elliptic equations and (1.1) is for presenting the main idea and techniques in their simplest form.

For given a shape regular triangulation $\mathcal{M}_{h}$ of $\bar{\Omega}$ with mesh size $h$, we denote

$$
V_{h}:=\left\{v_{h} \in H^{1}(\Omega):\left.v_{h}\right|_{\tau} \in P_{1}(\tau) \forall \tau \in \mathcal{M}_{h}\right\}
$$

the space of all continuous, piecewise linear finite element functions corresponding to $\mathcal{M}_{h}$. Here $P_{1}$ denotes the set of polynomials with degree at most one. The finite element solution $u_{h} \in V_{h}$ satisfies

$$
a\left(u_{h}, v_{h}\right)=f\left(v_{h}\right)+g\left(v_{h}\right), \quad \forall v \in H^{1}(\Omega) .
$$

\footnotetext{
${ }^{*}$ Received August 17, 2018 / Revised version received May 15, 2019 / Accepted November 28, 2019 / Published online January 6, 2020 /
} 
It is well known that there are many superclose and superconvergent results for Dirichlet boundary problems $[13,16,18,19,22]$. The convergence analysis is for uniform grids or patch symmetric grids at first. However, since it is difficult to construct uniform grids on unstructured domains and the grids produced by grid generation algorithms are a small perturbation of uniform grids in the most region of the domain, one considered the so-called mildly structured grids where an $O\left(h^{1+\alpha}\right)$ approximate parallelogram property is satisfied for pairs of adjacent triangles in most parts of $\Omega$ except for a region of size $O\left(h^{2 \sigma}\right)[6,7,16,18]$. Two finite element functions vanishing on $\partial \Omega$, the continuous linear finite element solution $u_{h}^{0}$ and the continuous linear nodal interpolation $u_{I}^{0}$ of $u^{0}$, are superclose in the sense that

$$
\left\|\nabla u_{h}^{0}-\nabla u_{I}^{0}\right\|_{H^{1}(\Omega)}=O\left(h^{1+\min (\alpha, 1-\sigma)}\right) .
$$

Here we assume that $u^{0}$ is the exact solution to the Dirichlet boundary problem. Based on the supercloseness, various post-processing techniques, such as the global $L^{2}$ projection $[6,8,11]$, the Zienkiewicz-Zhu (ZZ) method [24,25], and the Polynomial Preserving Recovery [13, 14, 23], have been proposed to produce a new approximation $R_{h}\left(u_{h}^{0}\right)$ of $\nabla u^{0}$, which is superconvergent in the sense that

$$
\left\|R_{h}\left(u_{h}^{0}\right)-\nabla u^{0}\right\|_{H^{1}(\Omega)}=O\left(h^{1+\min (\alpha, 1-\sigma)}\right) .
$$

Based on the superconvergence results, an asymptotically exact error estimator can be constructed $[7,16]$. In the last decade the convergence proof for Dirichlet boundary problem has been well established. By contrast, there are only a few superconvergent works on the Robin boundary problem. [9] considered the Robin boundary condition and proved the superconvergent rate of $O\left(h^{3 / 2}\right)$. [3] considered the case of Neumann boundary and $\alpha=1$ (i.e. each of the "good" pairs of triangles forms an $O\left(h^{2}\right)$ approximate parallelograms) and proved the superconvergent rate of $O\left(h^{2-\sigma}|\log h|^{\frac{1}{2}}\right)$.

In this work, we investigate the superconvergence property of the method (1.2) when being post-processed by the polynomial preserving recovery (PPR) for the Robin boundary problem. PPR was proposed by Zhang and Naga [23] in 2004 and has been successfully applied to finite element methods. COMSOL Multiphysics adopted PPR as a post-processing tool since 2008, see [1]. One important feature of PPR is its superconvergence property for the recovered gradient. To learn more about PPR, readers are referred to $[13,16,20,21]$. Some theoretical results about recovery techniques and recovery-type error estimators can be found in $[4,12,18,19,22]$.

We first extend the definition of mildly structured grids to the boundary by assuming that the two triangles associated to a "good" boundary node are $O\left(h^{1+\alpha}\right)$ approximate congruent triangles and the number of "bad" boundary nodes is of order $O\left(h^{-2 \mu}\right)$ for some $0 \leq \mu<\frac{1}{2}$. Secondly, we prove the following supercloseness result:

$$
\left\|u_{h}-u_{I}\right\|_{H^{1}(\Omega)}=O\left(h^{1+\min (\alpha, 1-\sigma)}+\min \left(h^{2-2 \mu} \mid \log h^{\frac{1}{2}}, h^{\frac{3}{2}}\right)\right),
$$

which improves the estimates of [3,9]. Denote $G_{h}: V_{h} \rightarrow V_{h} \times V_{h}$ as the gradient recovery operator from PPR. Thirdly, we obtain the following estimate:

$$
\left\|\nabla u-G_{h} u_{h}\right\|_{L^{2}(\Omega)} \lesssim h^{1+\min \{\alpha, 1-\sigma\}}+\min \left(h^{2-2 \mu}|\log h|^{\frac{1}{2}}, h^{3 / 2}\right) .
$$

Based on the superconvergent result, we define an asymptotically exact a posteriori error estimator $\left\|G_{h} u_{h}-\nabla u_{h}\right\|_{L^{2}(\Omega)}$. Readers are referred to $[2,5,10,15]$ for further theoretical results about recovery techniques and recovery-type error estimators. 
The remainder of this paper is organized as follows: some notations and the mesh constraints are introduced in Section 2. In Section 3, we prove the supercloseness between the interpolant and the finite element solution to the Robin boundary problem (1.1). In Section 4, we prove the superconvergence property of $G_{h}$ in the Sobolev space $H^{3}(\Omega)$ and define a posteriori error estimator. Finally, in Section 5 we verify the sharpness of our estimates by simulating some model problems on several specially designed meshes.

Throughout the paper, $C$ is used to denote a generic positive constant which is independent of $h, f$ and $g$. We also use the shorthand notation $A \lesssim B$ and $A \gtrsim B$ for the inequality $A \leq C B$ and $A \geq C B$, respectively. $A \approx B$ is a shorthand notation for the statement $A \lesssim B$ and $B \lesssim A$.

\section{Preliminaries}

The symbols $(\cdot, \cdot)_{Q}$ and $\langle\cdot, \cdot\rangle_{\Sigma}$ for $\Sigma=\partial Q$ denote the $L^{2}$-inner products on $L^{2}(Q)$ and $L^{2}(\Sigma)$ spaces, respectively. For simplicity, denote by $(\cdot, \cdot):=(\cdot, \cdot)_{\Omega},\langle\cdot, \cdot\rangle:=\langle\cdot, \cdot\rangle_{\partial \Omega},\|\cdot\|_{j}:=\|\cdot\|_{H^{j}(\Omega)}$, and $|\cdot|_{j}:=|\cdot|_{H^{j}(\Omega)}$. Throughout this paper, the norm $\|\cdot\|_{H^{s}(\Gamma)}$ (seminorm $|\cdot|_{H^{s}(\Gamma)}$ ) on the boundary of the polygon $\Omega$ are interpreted as the square root of the sum of squares of the $H^{s}$-norms (seminorms) on each sides of the polygon.

Let $\mathcal{E}_{h}$ be the set of all edges of $\mathcal{M}_{h}$ and $\mathcal{N}_{h}$ be the set of all nodal points. For any $\tau \in \mathcal{M}_{h}$, we denote by $h_{\tau}$ its diameter and by $|\tau|$ its area. Similarly, for each edge $e \in \mathcal{E}_{h}$, define $h_{e}:=\operatorname{diam}(e)$. Let $h=\max _{\tau \in \mathcal{M}_{h}} h_{\tau}$. Assume that $h_{\tau} \approx h$. We denote all the boundary edges by $\mathcal{E}_{h}^{B}:=\left\{e \in \mathcal{E}_{h}: e \subset \Gamma\right\}$ and the interior edges by $\mathcal{E}_{h}^{I}:=\mathcal{E}_{h} \backslash \mathcal{E}_{h}^{B}$. Denote by $\mathcal{N}_{h}^{B}$ the nodes on the physical boundary $\Gamma$.

Following the discussion in [6], We introduce some definitions regarding meshes at first. For an interior edge $e \in \mathcal{E}_{h}^{I}$, let $\tau_{e}$ and $\tau_{e}^{\prime}$ be two elements sharing $e$ and denote by $\Omega_{e}=\tau_{e} \cup \tau_{e}^{\prime}$, see Figures 2.1-2.2. For an element $\tau \subset \Omega_{e}$, denote by $\theta_{e}$ the angle opposite of the edge $e$ in $\tau$, denote by $\mathbf{t}_{e}$ the unit tangent vector of $e$ with counterclockwise orientation and $\mathbf{n}_{e}$, the unit outward normal vector of $e$. Denote by $h_{e}, h_{e+1}, h_{e-1}$ denote the lengths of the three edges of $\tau_{e}$, respectively, where the subscript $e+1$ or $e-1$ is for orientation. We emphasize that all triangles in $\mathcal{M}_{h}$ are orientated counterclockwise. An index ' is added for the corresponding quantities in $\tau_{e}^{\prime}$. Notice that $\mathbf{n}_{e}=-\mathbf{n}_{e}^{\prime}$ and $\mathbf{t}_{e}=-\mathbf{t}_{e}^{\prime}$ due to the orientation.

Let $e \in \mathcal{E}_{h}^{I}$ be an interior edge. Recall that $\Omega_{e}$, the patch of $e$, consists of two adjacent triangles sharing $e$. We say that $\Omega_{e}$ is an $\epsilon$ approximate parallelogram if the lengths of any two opposite edges differ by at most $\epsilon$, that is

$$
\left|h_{e-1}-h_{e-1}^{\prime}\right|+\left|h_{e+1}-h_{e+1}^{\prime}\right| \leq \epsilon
$$

In general, the patch $\Omega_{e}$ is set to be an $O\left(h^{1+\alpha}\right)$ approximate parallelogram in the supercenvergence analysis for Dirichlet boundary problems. However, for Robin boundary condition, an additional restriction on elements pairs with common nodes in $\mathcal{N}_{h}^{B}$ is necessary. For a node $z \in \mathcal{N}_{h}^{B}$ (cf. Fig. 2.2), let $e, e^{\prime} \in \mathcal{E}_{h}^{B}$ be the the edges sharing $z$. We say that $\tau_{e}$ and $\tau_{e^{\prime}}$ are $\varepsilon$ approximate congruent triangles if

$$
\left|h_{e-1}-h_{e^{\prime}-1}\right|+\left|h_{e+1}-h_{e^{\prime}+1}\right|+\left|h_{e}-h_{e^{\prime}}\right| \leq \epsilon .
$$

Clearly, for an interior edge $e$, if $\Omega_{e}$ is an $\epsilon$ approximate parallelogram, the two triangles in $\Omega_{e}$ are $\varepsilon$ approximate congruent triangles.

In order to deal with the Robin boundary condtion, we introduce the following mesh condition $(\alpha, \sigma, \mu)$ which is a modification of the usual condition $(\alpha, \sigma)$, see $[6,18]$. 


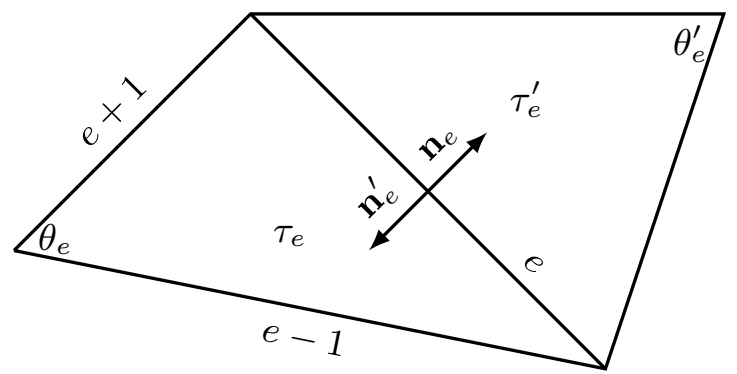

Fig. 2.1. Notation in the patch $\Omega_{e}$.

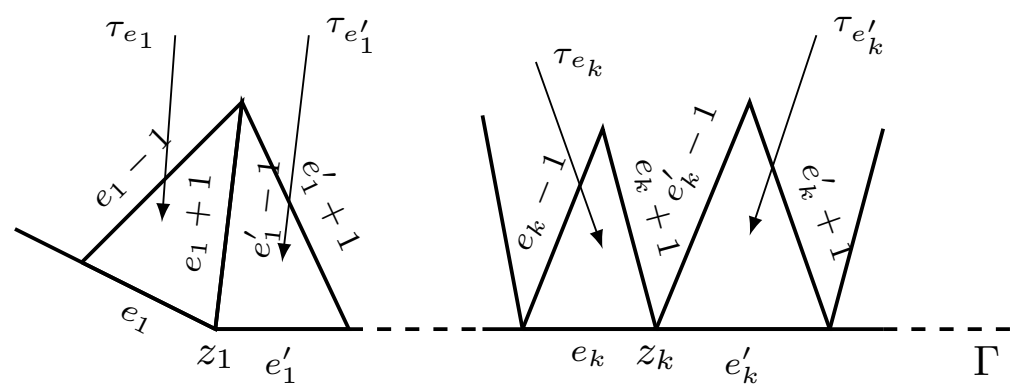

Fig. 2.2. Notation in the boundary elements.

Definition 2.1. The triangulation $\mathcal{M}_{h}$ is said to satisfy condition $(\alpha, \sigma, \mu)$ if $\mathcal{E}_{h}^{I}$ can be divided into two parts $\mathcal{E}_{1, h}^{I} \oplus \mathcal{E}_{2, h}^{I}, \mathcal{N}_{h}^{B}$ can be divided into two parts $\mathcal{N}_{1, h}^{B} \oplus \mathcal{N}_{2, h}^{B}$ and there exists constants $\alpha \geq 0,0 \leq \sigma<1$ and $0 \leq \mu<\frac{1}{2}$ such that

(i) for $e \in \mathcal{E}_{1, h}^{I}$, the patch $\Omega_{e}$ is an $O\left(h^{1+\alpha}\right)$ approximate parallelogram;

(ii) for $z \in \mathcal{N}_{1, h}^{B}$ shared by $e, e^{\prime} \in \mathcal{E}_{h}^{B}$, the triangles $\tau_{e}$ and $\tau_{e^{\prime}}$ are $O\left(h^{1+\alpha}\right)$ approximate congruent triangles;

(iii) the number of edges in $\mathcal{E}_{2, h}^{I}$ satisfies $\# \mathcal{E}_{2, h}^{I}=O\left(h^{-2 \sigma}\right)$;

(iv) the number of nodes in $\mathcal{N}_{2, h}^{B}$ satisfies $\# \mathcal{N}_{2, h}^{B}=O\left(h^{-2 \mu}\right)$.

Remark 2.1. (a) Bank and Xu proposed some mesh conditions [3, Definition 2.4] for a superconvergence analysis for the Neumann boundary condition. Our conditions (i) and (iii) are similar to the corresponding conditions in [3] (or more precisely, the same as the mildly structured grids defined in the reference [18]), while the conditions (ii) and (iv) are new, which are weaker than the corresponding condition 2 in [3, Definition 2.4]. In fact, [3] assumed that the triangles associated with each node $z \in \mathcal{N}_{1, h}^{B}$ form an $O\left(h^{2}\right)$ approximate parallelogram and that $\# \mathcal{N}_{2, h}^{B}=O(1)$. Therefore, our conditions actually define "mildly structured grids" up to the boundary and are more practical.

(b) Although one can still get results of superconvergence for some meshes not satisfying the restriction (i), such as Chevron pattern uniform mesh, we construct in Section 5 a class of special grids to show that the restriction " $h^{1+\alpha}$ approximate parallelogram" is necessary.

(c) However, since unlike the case of homogenous Dirichlet boundary conditions, $u_{h}-u_{I} \neq 0$ on $\Gamma$, we need the assumption (ii) for elements with one edge on $\Gamma$. Here $u_{I}$ is the linear 
interpolant of $u$. Note that whether the additional restriction (ii) in 2.1 is just technique for theoretical purpose or not is still an open problem. But if $\mathcal{E}_{2, h}^{I}=\emptyset$, we have $\mathcal{N}_{2, h}^{B}$ and the restriction (ii) can be removed, because, for any node $z \in \mathcal{N}_{h}^{B}$, all the triangles sharing the node $z$ are approximate congruent triangles. In others words, the condition $(\alpha, 0,0)$ is equivalent to classical condition $(\alpha, 0)$ and therefore, the results holds under the condition $(\alpha, 0)$ as well.

(d) The restrictions (iii) and (iv) in 2.1 means that $\left(\mathcal{E}_{h}^{I}, \mathcal{N}_{h}^{B}\right)$ can be grouped into "good" $\left(\mathcal{E}_{1, h}^{I}, \mathcal{N}_{1, h}^{B}\right)$ and "bad" $\left(\mathcal{E}_{2, h}^{I}, \mathcal{N}_{2, h}^{B}\right)$, respectively. The numbers of bad edges and bad boundary nodes are much smaller than those of good ones, respectively, where the ratios are

$$
\frac{\# \mathcal{E}_{2, h}^{I}}{\# \mathcal{E}_{1, h}^{I}} \lesssim \frac{h^{-2 \sigma}}{h^{-2}}=h^{2-2 \sigma}, \quad \frac{\# \mathcal{N}_{2, h}^{B}}{\# \mathcal{N}_{1, h}^{B}} \lesssim \frac{h^{-2 \mu}}{h^{-1}}=h^{1-2 \mu} .
$$

\section{Supercloseness Between the FE Solution and the Interpolant}

We investigate the supercloseness between the FE solution $u_{h}$ and the linear interpolant $u_{I}$ of $u$. Some special arguments are needed to establish the desired superclose result, because $u_{h}$ is not equal to $u_{I}$ on the physical boundary, that is $\left.\left(u_{h}-u_{I}\right)\right|_{\Gamma} \neq 0$.

We introduce the quadratic interpolant $\phi_{Q}=\Pi_{Q} \phi$ of $\phi$ based on nodal values and moment conditions on edges,

$$
\left(\Pi_{Q} \phi\right)(z)=\phi(z), \quad \int_{e} \Pi_{Q} \phi=\int_{e} \phi, \quad \forall z \in \mathcal{N}_{h}, e \in \mathcal{E}_{h} .
$$

The following fundamental identity for $v_{h} \in P_{1}(\tau)$ has been proved in [6]:

$$
\int_{\tau} \nabla\left(\phi-\phi_{I}\right) \cdot \nabla v_{h}=\sum_{e \in \partial \tau}\left(\beta_{e} \int_{e} \frac{\partial^{2} \phi_{Q}}{\partial \mathbf{t}_{e}^{2}} \frac{\partial v_{h}}{\partial \mathbf{t}_{e}}+\gamma_{e} \int_{e} \frac{\partial^{2} \phi_{Q}}{\partial \mathbf{t}_{e} \partial \mathbf{n}_{e}} \frac{\partial v_{h}}{\partial \mathbf{t}_{e}}\right),
$$

where

$$
\beta_{e}=\frac{1}{12} \cot \theta_{e}\left(h_{e+1}^{2}-h_{e-1}^{2}\right), \quad \gamma_{e}=\frac{1}{3} \cot \theta_{e}|\tau|,
$$

and $\phi_{I} \in P_{1}(\tau)$ is the linear interpolant of $\phi$ on $\tau$.

Lemma 3.1. We denote $\mathbf{m}_{e}$ by $\mathbf{t}_{e}$ or $\mathbf{n}_{e}$. Assume that $\mathcal{M}_{h}$ satisfies the condition $(\alpha, \sigma, \mu)$. Then we have the following estimates:

$$
\begin{array}{lll}
\left|\beta_{e}\right|+\left|\beta_{e}^{\prime}\right| \lesssim h^{2}, & \left|\gamma_{e}\right|+\left|\gamma_{e}^{\prime}\right| \lesssim h^{2}, & e \in \mathcal{E}_{h}^{I}, \\
\left|\beta_{e}-\beta_{e}^{\prime}\right| \lesssim h^{2+\alpha}, & \left|\gamma_{e}-\gamma_{e}^{\prime}\right| \lesssim h^{2+\alpha}, & e \in \mathcal{E}_{1, h}^{I}, \\
\left|\beta_{e}\right| \lesssim h^{2}, & \left|\gamma_{e}\right| \lesssim h^{2}, & e \in \mathcal{E}_{h}^{B}, \\
\left|\beta_{e}-\beta_{e}^{\prime}\right| \lesssim h^{2+\alpha}, & \left|\gamma_{e}-\gamma_{e}^{\prime}\right| \lesssim h^{2+\alpha}, & e \cap e^{\prime}=z \in \mathcal{N}_{1, h}^{B}, \quad e, e^{\prime} \in \mathcal{E}_{h}^{B} .
\end{array}
$$

Moreover, for any $e \in \mathcal{E}_{h}$,

$$
\begin{aligned}
& \int_{e} \frac{\partial^{2} \phi}{\partial \mathbf{t}_{e} \partial \mathbf{m}_{e}} \frac{\partial v_{h}}{\partial \mathbf{t}_{e}} \lesssim\left(|\phi|_{H^{3}\left(\tau_{e}\right)}+h^{-1}|\phi|_{H^{2}\left(\tau_{e}\right)}\right)\left\|\nabla v_{h}\right\|_{L^{2}\left(\tau_{e}\right)}, \\
& \int_{e} \frac{\partial^{2} \phi}{\partial \mathbf{t}_{e} \partial \mathbf{m}_{e}} \frac{\partial v_{h}}{\partial \mathbf{t}_{e}} \lesssim|\phi|_{W^{2, \infty}(\Omega)}\left\|\nabla v_{h}\right\|_{L^{2}\left(\tau_{e}\right)}, \\
& \int_{e} \frac{\partial^{2}\left(\phi-\phi_{Q}\right)}{\partial \mathbf{t}_{e} \partial \mathbf{m}_{e}} \frac{\partial v_{h}}{\partial \mathbf{t}_{e}} \lesssim|\phi|_{H^{3}\left(\tau_{e}\right)}\left\|\nabla v_{h}\right\|_{L^{2}\left(\tau_{e}\right)} .
\end{aligned}
$$


See $[6,7]$ for the proof.

Throughout the paper, we assume that $c \geq c_{0}>0, q \in W^{2, \infty}(\Gamma)(q \geq 0), f \in H^{1}(\Omega)$, $g \in H^{2}(\Gamma)$, and the exact solution $u \in H^{3}(\Omega) \cap W^{2, \infty}(\Omega)$.

Lemma 3.2. Assume that $\mathcal{M}_{h}$ satisfies the condition $(\alpha, \sigma, \mu)$. Then for any $v_{h} \in V_{h}$, we have

$$
\left|\int_{\Omega} \nabla\left(u-u_{I}\right) \cdot \nabla v_{h}\right| \lesssim\left(h^{1+\min (\alpha, 1-\sigma)}+\min \left(h^{2-2 \mu} \mid \log h^{\frac{1}{2}}, h^{\frac{3}{2}}\right)\right)\left\|v_{h}\right\|_{1} C_{u, g, q},
$$

where $C_{u, g, q}=\|u\|_{3}+|u|_{W^{2, \infty}(\Omega)}+\|g\|_{H^{2}(\Gamma)}+\|q\|_{W^{2, \infty}(\Gamma)}\|u\|_{H^{2}(\Gamma)}+\|u\|_{H^{3}(\Gamma)}$.

Proof. From (3.2), we have

$$
\int_{\Omega} \nabla\left(u-u_{I}\right) \cdot \nabla v_{h}=\sum_{\tau \in \mathcal{M}_{h}} \sum_{e \subset \partial \tau}\left(\beta_{e} \int_{e} \frac{\partial^{2} u_{Q}}{\partial \mathbf{t}_{e}^{2}} \frac{\partial v_{h}}{\partial \mathbf{t}_{e}}+\gamma_{e} \int_{e} \frac{\partial^{2} u_{Q}}{\partial \mathbf{t}_{e} \partial \mathbf{n}_{e}} \frac{\partial v_{h}}{\partial \mathbf{t}_{e}}\right)=: I_{1}+I_{2},
$$

where

$$
\begin{aligned}
I_{1}=\sum_{e \in \mathcal{E}_{h}^{I}} & \left(\left(\beta_{e}-\beta_{e}^{\prime}\right) \int_{e} \frac{\partial^{2} u}{\partial \mathbf{t}_{e}^{2}} \frac{\partial v_{h}}{\partial \mathbf{t}_{e}}+\left(\gamma_{e}-\gamma_{e}^{\prime}\right) \int_{e} \frac{\partial^{2} u}{\partial \mathbf{t}_{e} \partial \mathbf{n}_{e}} \frac{\partial v_{h}}{\partial \mathbf{t}_{e}}\right. \\
& +\beta_{e} \int_{e} \frac{\partial^{2}\left(u_{Q}-u\right)}{\partial \mathbf{t}_{e}^{2}} \frac{\partial v_{h}}{\partial \mathbf{t}_{e}}+\gamma_{e} \int_{e} \frac{\partial^{2}\left(u_{Q}-u\right)}{\partial \mathbf{t}_{e} \partial \mathbf{n}_{e}} \frac{\partial v_{h}}{\partial \mathbf{t}_{e}} \\
& \left.+\beta_{e}^{\prime} \int_{e} \frac{\partial^{2}\left(u-u_{Q}\right)}{\partial \mathbf{t}_{e}^{2}} \frac{\partial v_{h}}{\partial \mathbf{t}_{e}}+\gamma_{e}^{\prime} \int_{e} \frac{\partial^{2}\left(u-u_{Q}\right)}{\partial \mathbf{t}_{e} \partial \mathbf{n}_{e}} \frac{\partial v_{h}}{\partial \mathbf{t}_{e}}\right), \\
I_{2}=\sum_{e \in \mathcal{E}_{h}^{B}} & \left(\beta_{e} \int_{e} \frac{\partial^{2} u}{\partial \mathbf{t}_{e}^{2}} \frac{\partial v_{h}}{\partial \mathbf{t}_{e}}+\gamma_{e} \int_{e} \frac{\partial^{2} u}{\partial \mathbf{t}_{e} \partial \mathbf{n}_{e}} \frac{\partial v_{h}}{\partial \mathbf{t}_{e}}\right. \\
& \left.+\beta_{e} \int_{e} \frac{\partial^{2}\left(u_{Q}-u\right)}{\partial \mathbf{t}_{e}^{2}} \frac{\partial v_{h}}{\partial \mathbf{t}_{e}}+\gamma_{e} \int_{e} \frac{\partial^{2}\left(u_{Q}-u\right)}{\partial \mathbf{t}_{e} \partial \mathbf{n}_{e}} \frac{\partial v_{h}}{\partial \mathbf{t}_{e}}\right) .
\end{aligned}
$$

We first estimate $I_{1}$ which is divided into $I_{1,1}$ and $I_{1,2}$, where

$$
\begin{aligned}
I_{1, j}= & \sum_{e \in \mathcal{E}_{j, h}^{I}}\left(\left(\beta_{e}-\beta_{e}^{\prime}\right) \int_{e} \frac{\partial^{2} u}{\partial \mathbf{t}_{e}^{2}} \frac{\partial v_{h}}{\partial \mathbf{t}_{e}}+\left(\gamma_{e}-\gamma_{e}^{\prime}\right) \int_{e} \frac{\partial^{2} u}{\partial \mathbf{t}_{e} \partial \mathbf{n}_{e}} \frac{\partial v_{h}}{\partial \mathbf{t}_{e}}\right. \\
& +\beta_{e} \int_{e} \frac{\partial^{2}\left(u_{Q}-u\right)}{\partial \mathbf{t}_{e}^{2}} \frac{\partial v_{h}}{\partial \mathbf{t}_{e}}+\gamma_{e} \int_{e} \frac{\partial^{2}\left(u_{Q}-u\right)}{\partial \mathbf{t}_{e} \partial \mathbf{n}_{e}} \frac{\partial v_{h}}{\partial \mathbf{t}_{e}} \\
& \left.+\beta_{e}^{\prime} \int_{e} \frac{\partial^{2}\left(u-u_{Q}\right)}{\partial \mathbf{t}_{e}^{2}} \frac{\partial v_{h}}{\partial \mathbf{t}_{e}}+\gamma_{e}^{\prime} \int_{e} \frac{\partial^{2}\left(u-u_{Q}\right)}{\partial \mathbf{t}_{e} \partial \mathbf{n}_{e}} \frac{\partial v_{h}}{\partial \mathbf{t}_{e}}\right) .
\end{aligned}
$$

By Lemma 3.1 and Hölder's inequality, we have

$$
\begin{aligned}
\left|I_{1,1}\right| & \lesssim \sum_{e \in \mathcal{E}_{1, h}^{I}}\left(\left(h^{2+\alpha}+h^{2}\right)|u|_{H^{3}\left(\tau_{e}\right)}+h^{1+\alpha}|u|_{H^{2}\left(\tau_{e}\right)}\right)\left\|\nabla v_{h}\right\|_{L^{2}\left(\tau_{e}\right)} \\
& \lesssim\left(h^{2}|u|_{3}+h^{1+\alpha}|u|_{2}\right)\left\|\nabla v_{h}\right\|_{0} .
\end{aligned}
$$

From Lemma 3.1 and noting that $\# \mathcal{E}_{2, h}^{I}=h^{-2 \sigma}$,

$$
\begin{aligned}
\left|I_{1,2}\right| & \lesssim \sum_{e \in \mathcal{E}_{2, h}^{I}} h^{2}|u|_{W^{2, \infty}\left(\tau_{e}\right)}\left\|\nabla v_{h}\right\|_{L^{2}\left(\tau_{e}\right)} \lesssim h^{2}\left(\# \mathcal{E}_{2, h}^{I}\right)^{\frac{1}{2}}|u|_{W^{2, \infty}(\Omega)}\left\|\nabla v_{h}\right\|_{0} \\
& \lesssim h^{2-\sigma}|u|_{W^{2, \infty}(\Omega)}\left\|\nabla v_{h}\right\|_{0} .
\end{aligned}
$$


Combining (3.12) and (3.13) yields

$$
\left|I_{1}\right| \lesssim\left(h^{1+\alpha}|u|_{2}+h^{2-\sigma}|u|_{W^{2, \infty}(\Omega)}+h^{2}|u|_{H^{3}(\Omega)}\right)\left\|\nabla v_{h}\right\|_{0} .
$$

We divide $I_{2}$ into two parts $I_{2, j}(j=1,2)$ to estimate, where

$$
\begin{aligned}
I_{2,1} & :=\sum_{e \in \mathcal{E}_{h}^{B}}\left(\beta_{e} \int_{e} \frac{\partial^{2}\left(u_{Q}-u\right)}{\partial \mathbf{t}_{e}^{2}} \frac{\partial v_{h}}{\partial \mathbf{t}_{e}}+\gamma_{e} \int_{e} \frac{\partial^{2}\left(u_{Q}-u\right)}{\partial \mathbf{t}_{e} \partial \mathbf{n}_{e}} \frac{\partial v_{h}}{\partial \mathbf{t}_{e}}\right), \\
I_{2,2} & :=\sum_{e \in \mathcal{E}_{h}^{B}}\left(\beta_{e} \int_{e} \frac{\partial^{2} u}{\partial \mathbf{t}_{e}^{2}} \frac{\partial v_{h}}{\partial \mathbf{t}_{e}}+\gamma_{e} \int_{e} \frac{\partial^{2} u}{\partial \mathbf{t}_{e} \partial \mathbf{n}_{e}} \frac{\partial v_{h}}{\partial \mathbf{t}_{e}}\right) .
\end{aligned}
$$

From Lemma 3.1, we can derive in analogy to (3.12),

$$
\begin{aligned}
\left|I_{2,1}\right| & \lesssim \sum_{e \in \mathcal{E}_{1, h}^{B}}\left(h^{1+\alpha}|u|_{H^{2}\left(\tau_{e}\right)}+\left(h^{2+\alpha}+h^{2}\right)|u|_{H^{3}\left(\tau_{e}\right)}\right)\left\|\nabla v_{h}\right\|_{L^{2}\left(\tau_{e}\right)} \\
& \lesssim\left(h^{1+\alpha}|u|_{2}+h^{2}|u|_{3}\right)\left\|\nabla v_{h}\right\|_{0} .
\end{aligned}
$$

Next we turn to the estimate $I_{2,2}$. First we have

$$
\begin{aligned}
I_{2,2} & \lesssim h^{2} \sum_{e \in \mathcal{E}_{h}^{B}}|u|_{W^{2, \infty}(\Omega)}\left\|\nabla v_{h}\right\|_{L^{2}\left(\tau_{e}\right)} \lesssim h^{2}\left(\# \mathcal{E}_{h}^{B}\right)^{\frac{1}{2}}|u|_{W^{2, \infty}(\Omega)}\left\|\nabla v_{h}\right\|_{0} \\
& \lesssim h^{\frac{3}{2}}|u|_{W^{2, \infty}(\Omega)}\left\|\nabla v_{h}\right\|_{0} .
\end{aligned}
$$

In the following we give another estimate of $I_{2,2}$ by using the assumption on the elements with sides on the boundary $\Gamma$. For $z \in \mathcal{N}_{h}^{B}$, let $e$ and $e^{\prime}$ be two edges in $\mathcal{E}_{h}^{B}$ sharing $z$ with counterclockwise orientation (cf. Figure 2.2). Denote by $\left[\beta_{e}\right]_{z}=\beta_{e^{\prime}}-\beta_{e}$ and $\left[\gamma_{e}\right]_{z}=\gamma_{e^{\prime}}-\gamma_{e}$. Let $\mathcal{N}_{h}^{V}$ be the set of vertices of the domain $\Omega$. By integration by parts, we have

$$
\begin{aligned}
I_{2,2}=-\sum_{e \in \mathcal{E}_{h}^{B}}\left(\beta_{e} \int_{e} \frac{\partial^{3} u}{\partial \mathbf{t}_{e}^{3}} v_{h}+\gamma_{e} \int_{e} \frac{\partial^{3} u}{\partial \mathbf{t}_{e}^{2} \partial \mathbf{n}_{e}} v_{h}\right) \\
\quad+\sum_{z \in \mathcal{N}_{1, h}^{B} \backslash \mathcal{N}_{h}^{V}}\left(\left[\beta_{e}\right]_{z} \frac{\partial^{2} u}{\partial \mathbf{t}_{e}^{2}}(z)+\left[\gamma_{e}\right]_{z} \frac{\partial^{2} u}{\partial \mathbf{t}_{e} \partial \mathbf{n}_{e}}(z)\right) v_{h}(z) \\
+\sum_{z \in \mathcal{N}_{2, h}^{B} \backslash \mathcal{N}_{h}^{V}}\left(\left[\beta_{e}\right]_{z} \frac{\partial^{2} u}{\partial \mathbf{t}_{e}^{2}}(z)+\left[\gamma_{e}\right]_{z} \frac{\partial^{2} u}{\partial \mathbf{t}_{e} \partial \mathbf{n}_{e}}(z)\right) v_{h}(z) \\
\quad+\sum_{z \in \mathcal{N}_{h}^{V}}\left(\beta_{e^{\prime}} \frac{\partial^{2} u}{\partial \mathbf{t}_{e^{\prime}}^{2}}(z)-\beta_{e} \frac{\partial^{2} u}{\partial \mathbf{t}_{e}^{2}}(z)+\gamma_{e^{\prime}} \frac{\partial^{2} u}{\partial \mathbf{t}_{e^{\prime}} \partial \mathbf{n}_{e^{\prime}}}(z)-\gamma_{e} \frac{\partial^{2} u}{\partial \mathbf{t}_{e} \partial \mathbf{n}_{e}}(z)\right) v_{h}(z) \\
=: I_{2,2,1}+I_{2,2,2}+I_{2,2,3}+I_{2,2,4} .
\end{aligned}
$$

Since $\frac{\partial u}{\partial \mathbf{n}}+q u=g$ on $\Gamma$, we obtain

$$
\begin{aligned}
I_{2,2,1} & \lesssim h^{2}\left(|u|_{H^{3}(\Gamma)}+\left|\frac{\partial u}{\partial \mathbf{n}}\right|_{H^{2}(\Gamma)}\right)\left\|v_{h}\right\|_{L^{2}(\Gamma)} \\
& \lesssim h^{2}\left(|u|_{H^{3}(\Gamma)}+|g|_{H^{2}(\Gamma)}+|q u|_{H^{2}(\Gamma)}\right)\left\|v_{h}\right\|_{0}^{\frac{1}{2}}\left\|v_{h}\right\|_{1}^{\frac{1}{2}} \\
& \lesssim h^{2}\left\|v_{h}\right\|_{1}\left(|g|_{H^{2}(\Gamma)}+\|q\|_{W^{2, \infty}(\Gamma)}|u|_{H^{2}(\Gamma)}+|u|_{H^{3}(\Gamma)}\right) .
\end{aligned}
$$


For any $w \in H^{1}([a, b])$, we have

$$
w^{2}(b)=\int_{a}^{b}\left(\frac{x-a}{b-a} w^{2}(x)\right)^{\prime} d x \lesssim \frac{1}{b-a}\|w\|_{L^{2}([a, b])}^{2}+(b-a)\|w\|_{L^{2}([a, b])}^{2},
$$

which implies

$$
\begin{aligned}
I_{2,2,2} \leq & \sum_{z \in \mathcal{N}_{1, h}^{B} \backslash \mathcal{N}_{h}^{V}}\left(\left|\left[\beta_{e}\right]_{z}\right|\left(h_{e}^{-\frac{1}{2}}|u|_{H^{2}(e)}+h_{e}^{\frac{1}{2}}|u|_{H^{3}(e)}\right)\right. \\
& \left.+\left|\left[\gamma_{e}\right]_{z}\right|\left(h_{e}^{-\frac{1}{2}}\left|\frac{\partial u}{\partial \mathbf{n}_{e}}\right|_{H^{1}(e)}+h_{e}^{\frac{1}{2}}\left|\frac{\partial u}{\partial \mathbf{n}_{e}}\right|_{H^{2}(e)}\right)\right) \\
& \cdot\left(h_{e}^{-\frac{1}{2}}\left\|v_{h}\right\|_{L^{2}(e)}+h_{e}^{\frac{1}{2}}\left|v_{h}\right|_{H^{1}(e)}\right) \\
\lesssim & \max _{z \in \mathcal{N}_{1, h}^{B} \backslash \mathcal{N}_{h}^{V}}\left(\left|\left[\beta_{e}\right]_{z}\right|+\left|\left[\gamma_{e}\right]_{z}\right|\right)\left(h^{-\frac{1}{2}}|u|_{H^{2}(\Gamma)}+h^{\frac{1}{2}}|u|_{H^{3}(\Gamma)}\right. \\
& \left.+h^{-\frac{1}{2}}\left|\frac{\partial u}{\partial \mathbf{n}}\right|_{H^{1}(\Gamma)}+h^{\frac{1}{2}}\left|\frac{\partial u}{\partial \mathbf{n}}\right|_{H^{2}(\Gamma)}\right) \cdot h^{-\frac{1}{2}}\left\|v_{h}\right\|_{L^{2}(\Gamma)} \\
\lesssim h^{1+\alpha} & \left(|u|_{H^{2}(\Gamma)}+h|u|_{H^{3}(\Gamma)}+|g|_{H^{1}(\Gamma)}+h|g|_{H^{2}(\Gamma)}\right. \\
& \left.+\|q\|_{W^{1, \infty}(\Gamma)}|u|_{H^{1}(\Gamma)}+h\|q\|_{W^{2, \infty}(\Gamma)}|u|_{H^{2}(\Gamma)}\right)\left\|v_{h}\right\|_{1},
\end{aligned}
$$

where we have used the second inequality in (3.7).

The following inequality [17] is going to be used to estimate $I_{2,2,3}+I_{2,2,4}$ :

$$
\left\|v_{h}\right\|_{L^{\infty}(\Omega)} \lesssim|\log h|^{\frac{1}{2}}\left\|v_{h}\right\|_{1} \quad \forall v_{h} \in V_{h} .
$$

Noting that $\# \mathcal{N}_{2, h}^{B}=h^{-2 \mu}$, we have

$$
\begin{aligned}
& I_{2,2,3}+I_{2,2,4} \\
\lesssim & h^{2} \sum_{z \in \mathcal{N}_{2, h}^{B} \cup \mathcal{N}_{h}^{V}}\left(\left|\frac{\partial^{2} u}{\partial \mathbf{t}_{e^{\prime}}^{2}}(z)\right|+\left|\frac{\partial^{2} u}{\partial \mathbf{t}_{e}^{2}}(z)\right|+\left|\frac{\partial^{2} u}{\partial \mathbf{t}_{e^{\prime}} \partial \mathbf{n}_{e^{\prime}}}(z)\right|+\left|\frac{\partial^{2} u}{\partial \mathbf{t}_{e} \partial \mathbf{n}_{e}}(z)\right|\right)\left\|v_{h}\right\|_{L^{\infty}(\Omega)} \\
\lesssim & h^{2}\left(\# \mathcal{N}_{2, h}^{B}+\# \mathcal{N}_{h}^{V}\right)\left(\|u\|_{H^{3}(\Gamma)}+\|g\|_{H^{2}(\Gamma)}+\|q\|_{W^{2, \infty}(\Gamma)}\|u\|_{H^{2}(\Gamma)}\right) \cdot|\log h|^{\frac{1}{2}}\left\|v_{h}\right\|_{H^{1}(\Omega)} \\
\lesssim & h^{2-2 \mu}|\log h|^{\frac{1}{2}}\left\|v_{h}\right\|_{1}\left(\|u\|_{H^{3}(\Gamma)}+\|g\|_{H^{2}(\Gamma)}+\|q\|_{W^{2, \infty}(\Gamma)}\|u\|_{H^{2}(\Gamma)}\right) .
\end{aligned}
$$

Using (3.14), (3.17), (3.18)-(3.22), we complete the proof of the lemma.

We can also obtain the supercloseness between $u_{h}$ and $u_{I}$ from Lemma 3.2.

Theorem 3.1. Assume that $\mathcal{M}_{h}$ satisfies the condition $(\alpha, \sigma, \mu)$. We have

$$
\left\|u_{h}-u_{I}\right\|_{1} \lesssim\left(h^{1+\min (\alpha, 1-\sigma)}+\min \left(h^{2-2 \mu}|\log h|^{\frac{1}{2}}, h^{\frac{3}{2}}\right)\right) C_{u, g, q} .
$$

Proof. Denote $v_{h}=u_{h}-u_{I}$. By the Galerkin orthogonality, we have

$$
\begin{aligned}
& \left\|u_{h}-u_{I}\right\|_{1}^{2} \lesssim a\left(u_{h}-u_{I}, v_{h}\right) \\
= & \left(\nabla\left(u-u_{I}\right), \nabla v_{h}\right)+\left(c\left(u-u_{I}\right), v_{h}\right)+\left\langle q\left(u-u_{I}\right), v_{h}\right\rangle .
\end{aligned}
$$


By the trace inequality,

$$
\begin{aligned}
\left|\left\langle q\left(u-u_{I}\right), v_{h}\right\rangle\right| & \leq\|q\|_{L^{\infty}(\Gamma)}\left\|u-u_{I}\right\|_{L^{2}(\Gamma)}\left\|v_{h}\right\|_{L^{2}(\Gamma)} \\
& \lesssim h^{2}\|q\|_{L^{\infty}(\Gamma)}\|u\|_{H^{2}(\Gamma)} \cdot\left\|v_{h}\right\|_{L^{2}(\Gamma)} .
\end{aligned}
$$

By combining Lemma 3.2, (3.24), (3.25), and the fact that $\left\|u-u_{I}\right\|_{0} \lesssim h^{2}\|u\|_{2}$, we complete the proof.

Remark 3.1. Our estimate of supercloseness holds on mildly structured grids up to the boundary. In particular, under the same conditions as [3, Theorem 3.1] (i.e. $q=0, g=0, \mu=$ $0, \alpha=1$ ) our Theorem 3.1 implies that

$$
\left\|u_{h}-u_{I}\right\|_{1} \lesssim\left(h^{2-\sigma}+h^{2}|\log h|^{\frac{1}{2}}\right)\left(\|u\|_{3}+|u|_{W^{2, \infty}(\Omega)}+|u|_{H^{3}(\Gamma)}\right) .
$$

When $\sigma>0$, our convergence order is $O\left(h^{2-\sigma}\right)$ since $h^{\sigma}|\log h|^{\frac{1}{2}} \rightarrow 0$ as $h \rightarrow 0^{+}$, while the order given by [3] is $O\left(h^{2-\sigma}|\log h|^{\frac{1}{2}}\right)$. On the other hand, our regularity requirement on $u$ is weaker than that of $[3]$.

\section{Superconvergence and the a Posteriori Error Estimator}

In this section, we introduce and analyze the polynomial preserving recovery method (PPR), which is applied to improve the gradient of the finite element solution.

We first recall the polynomial preserving method (PPR) [13]. Define $G_{h}: C(\bar{\Omega}) \mapsto V_{h} \times V_{h}$ as the gradient recovery operator on the finite element space in the following way: Given a node $z \in \mathcal{N}_{h}$, select an element patch $\omega_{z}$. We then denote all nodes on $\omega_{z}$ (including $z$ ) as $z_{j}, j=1,2, \cdots, n(\geq 6)$ and use values of $w \in C(\bar{\Omega})$ at these sampling points to fit a quadratic polynomial in the least squares sense

$$
\sum_{j=1}^{n}\left(p_{2}-w\right)^{2}\left(z_{j}\right)=\min _{q \in P_{2}} \sum_{j=1}^{n}(q-w)^{2}\left(z_{j}\right) .
$$

Here $P_{2}\left(\omega_{z}\right)$ is the set of continuous, piecewise quadratic polynomial functions defined on $\omega_{z}$. The value of the recovered gradient of $w$ at $z$ is then defined as

$$
G_{h} w(z)=\left(\nabla p_{2}\right)(z)
$$

The reader is referred to [13, Theorem 2.3] for a practical sufficient condition so that the above least squares fitting procedure has a unique solution. We shall use the PPR method to improve the numerical solutions.

Some properties of the PPR operator should be shown:

(i) $\left\|G_{h} v_{h}\right\|_{0} \lesssim\left\|\nabla v_{h}\right\|_{0} \quad \forall v_{h} \in V_{h}$.

(ii) For any nodal point $z,\left(G_{h} p\right)(z)=\nabla p(z)$ if $p \in P_{j}\left(\omega_{z}\right), j=1,2$.

(iii) $G_{h} w=G_{h} I_{h}^{j} w \quad \forall w \in C(\bar{\Omega}), j=1,2$.

Here $I_{h}^{1} w$ and $I_{h}^{2} w$ are the linear nodal value interpolant and quadratic nodal value interpolant of $w$, respectively. The reader is referred to $[13,16,21,23]$ for more details of these properties. 
We decompose the error into

$$
\nabla u-G_{h} u_{h}=\nabla u-G_{h} u+G_{h}\left(u_{I}-u_{h}\right) .
$$

Note that $G_{h} u=G_{h} u_{I}$ since $u_{I}=u$ at all vertices and the recovery operator $G_{h}$ is completely determined by nodal values of $u$. From (i), we have $\left\|G_{h}\left(u_{I}-u_{h}\right)\right\|_{0} \lesssim\left\|\nabla\left(u_{I}-u_{h}\right)\right\|_{0}$. It remains to estimate $\left\|\nabla u-G_{h} u\right\|_{0}$.

Lemma 4.1. For any element $\tau \in \mathcal{M}_{h}$ and any function $w \in H^{3}(\tilde{\tau})$,

$$
\left\|G_{h} w-\nabla w\right\|_{L^{2}(\tau)} \lesssim h^{2}\|w\|_{H^{3}(\tilde{\tau})}
$$

where $\tilde{\tau}=\bigcup\left\{\omega_{z}: z \in \mathcal{N}_{h} \cap \tau\right\}$.

Proof. The property (iii) implies

$$
\left\|G_{h} w-\nabla w\right\|_{L^{2}(\tau)}=\left\|G_{h} I_{h}^{2} w-\nabla w\right\|_{L^{2}(\tau)} .
$$

For any $\eta \in P_{2}(\tilde{\tau})$, it is clear that $G_{h} \eta=\nabla \eta$ in $\tau$ by the property (ii) and the fact that $G_{h} \eta \in V_{h} \times V_{h}$ and $\nabla \eta \in P_{1}(\tilde{\tau}) \times P_{1}(\tilde{\tau})$. Then we have

$$
\begin{aligned}
\left\|G_{h} I_{h}^{2} w-\nabla w\right\|_{L^{2}(\tau)} & =\left\|G_{h}\left(I_{h}^{2} w-\eta\right)-\nabla(w-\eta)\right\|_{L^{2}(\tau)} \\
& \leq\left\|G_{h}\left(I_{h}^{2} w-\eta\right)\right\|_{L^{2}(\tau)}+\|\nabla(w-\eta)\|_{L^{2}(\tau)} .
\end{aligned}
$$

By the definition and properties of $G_{h}$,

$$
\begin{aligned}
& \left\|G_{h}\left(I_{h}^{2} w-\eta\right)\right\|_{L^{2}(\tau)} \\
\lesssim & h \max _{z \in \mathcal{N}_{h} \cap \tau}\left|G_{h}\left(I_{h}^{2} w-\eta\right)(z)\right| \lesssim h\left\|\nabla\left(I_{h}^{2} w-\eta\right)\right\|_{L^{\infty}(\tilde{\tau})} \\
\lesssim & \left\|\nabla\left(I_{h}^{2} w-\eta\right)\right\|_{L^{2}(\tilde{\tau})} \lesssim\left\|\nabla\left(I_{h}^{2} w-w\right)\right\|_{L^{2}(\tilde{\tau})}+\|\nabla(w-\eta)\|_{L^{2}(\tilde{\tau})}
\end{aligned}
$$

By combining (4.5)-(4.7), we have

$$
\left\|G_{h} I_{h}^{2} w-\nabla w\right\|_{L^{2}(\tau)} \lesssim \inf _{\eta \in P_{2}(\tilde{\tau})}\|\nabla(w-\eta)\|_{L^{2}(\tilde{\tau})}+\left\|\nabla\left(I_{h}^{2} w-w\right)\right\|_{L^{2}(\tilde{\tau})} .
$$

Using the Bramble-Hilbert lemma and the scaling argument, we have

$$
\inf _{\eta \in P_{2}(\tilde{\tau})}\|\nabla(w-\eta)\|_{L^{2}(\tilde{\tau})} \lesssim h^{2}\|w\|_{H^{3}(\tilde{\tau})},
$$

and from the approximation theory

$$
\left\|\nabla\left(I_{h}^{2} w-w\right)\right\|_{L^{2}(\tilde{\tau})} \lesssim h^{2}\|w\|_{H^{3}(\tilde{\tau})} .
$$

The proof is completed by combining (4.5) with (4.8)-(4.10).

We remark that similar results as the above lemma are proved in [16,18]. Using Lemma 4.1, we obtain the following superconvergence property of $G_{h}$ in $H^{3}(\Omega)$ space for the linear element.

Theorem 4.1. We have the following estimate:

$$
\left\|G_{h} u-\nabla u\right\|_{0} \lesssim h^{2}\|u\|_{3}
$$


Proof. From Lemma 4.1, we have

$$
\left\|G_{h} u-\nabla u\right\|_{0}=\left(\sum_{\tau \in \mathcal{M}_{h}}\left\|G_{h} u-\nabla u\right\|_{L^{2}(\tau)}^{2}\right)^{\frac{1}{2}} \lesssim h^{2}\left(\sum_{\tau \in \mathcal{M}_{h}}\|u\|_{H^{3}(\tilde{\tau})}^{2}\right)^{\frac{1}{2}} \lesssim h^{2}\|u\|_{3} .
$$

We obtain the superconvercengce property of the linear FE solution post-processed by the PPR operator, which can be proved by combining (4.3), Theorems 3.1 and 4.1 .

Theorem 4.2. Assume that $\mathcal{M}_{h}$ satisfies condition $(\alpha, \sigma, \mu)$. Let $u$ and $u_{h}$ be the solutions to (1.1) and (1.2), respectively. We have

$$
\left\|G_{h} u_{h}-\nabla u\right\|_{0} \lesssim\left(h^{1+\min (\alpha, 1-\sigma)}+\min \left(h^{2-2 \mu}|\log h|^{\frac{1}{2}}, h^{\frac{3}{2}}\right)\right) C_{u, g, q}
$$

With preparation from the previous work, it is now straightforward to prove the asymptotic exactness of an error estimator based on the recovery operator $G_{h}$. The global error estimator is naturally defined by

$$
\eta_{h}=\left\|G_{h} u_{h}-\nabla u_{h}\right\|_{0}
$$

Theorem 4.3. Let $u_{h}$ be the linear finite element approximation of $u$. Assume that $\mathcal{M}_{h}$ satisfies Condition $(\alpha, \sigma, \mu)$. Furthermore, assume that $h \lesssim\left\|\nabla\left(u-u_{h}\right)\right\|_{0}$. Then

$$
\left|\frac{\eta_{h}}{\left\|\nabla\left(u-u_{h}\right)\right\|_{0}}-1\right| \lesssim h^{\rho}+\min \left(h^{1-2 \mu} \mid \log h^{\frac{1}{2}}, h^{\frac{1}{2}}\right), \quad \rho=\min (\alpha, 1-\sigma) .
$$

\section{Numerical Examples}

In this section we present experiments to study the supercloseness and superconvergence rates on different meshes in $H^{1}$-seminorms as well as the asymptotically exactness of the a posteriori error estimator based on the recovery operator $G_{h}$.

we solve $-\Delta u+u=f$ with the Robin boundary condition $\frac{\partial u}{\partial \mathbf{n}}+u=g$, where $f$ and $g$ are chosen such that the sulotion is $u=\sin (\pi(x+y))$.

We consider the supercloseness property on the domain $\bar{\Omega}=[0,1] \times[0,1]$. We first consider discretizations performed on meshes consisting of $O\left(h^{1+\alpha}\right)$ approximate parallelograms. We divide $\bar{\Omega}$ into $\frac{n}{2} \times n$ equivalent rectanges which containing four types of triangles (cf. Fig. 5.1) , where $h=1 / n$ and $2 \tilde{h}+\tilde{h}^{1+\alpha}=2 h$, for even integer $n$. Clearly, $\frac{2}{3} h \leq \tilde{h} \leq h$. Fig. 5.2
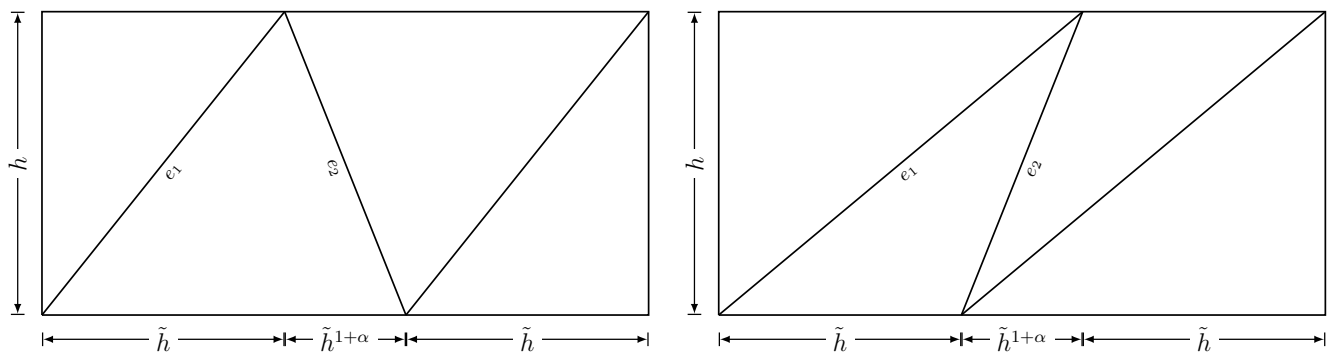

Fig. 5.1. The cells constituting the finite element grids. 
plots the meshes for $n=4,32$ and $\alpha=0, \frac{1}{2}$, respectively. For any $e \in \mathcal{E}_{h}^{I}$, denote by $\epsilon_{e}=$ $\left|h_{e-1}-h_{e-1}^{\prime}\right|+\left|h_{e+1}-h_{e+1}^{\prime}\right|$. For $e_{1}$ in Fig. 5.1, we have

$$
\begin{aligned}
\left(\frac{2}{3} h\right)^{1+\alpha} \leq \epsilon_{e_{1}} & =\tilde{h}^{1+\alpha}+\left(h^{2}+\tilde{h}^{2+2 \alpha}\right)^{\frac{1}{2}}-h=\tilde{h}^{1+\alpha}+\frac{\tilde{h}^{2+2 \alpha}}{\left(h^{2}+\tilde{h}^{2+2 \alpha}\right)^{\frac{1}{2}}+h} \\
& \leq \tilde{h}^{1+\alpha}+\frac{\tilde{h}^{2+2 \alpha}}{2 h} \leq \frac{3}{2} \tilde{h}^{1+\alpha} \leq \frac{3}{2} h^{1+\alpha} .
\end{aligned}
$$

Moreover, it is clear that $\epsilon_{e_{2}}=0$ for $e_{2}$ in Fig. 5.1. As a matter of fact, it can be shown that for every $e \in \mathcal{E}_{h}^{I}, \Omega_{e}$ is an $O\left(h^{1+\alpha}\right)$ approximate parallelogram. Similarly, for any $z \in \mathcal{N}_{h}^{B} \backslash \mathcal{N}_{h}^{V}$ shared by $e, e^{\prime} \in \mathcal{E}_{h}^{B}$, the triangles $\tau_{e}$ and $\tau_{e^{\prime}}$ are $O\left(h^{1+\alpha}\right)$ approximate congruent triangles. That is, the meshes satisfy Condition $(\alpha, 0,0)$.
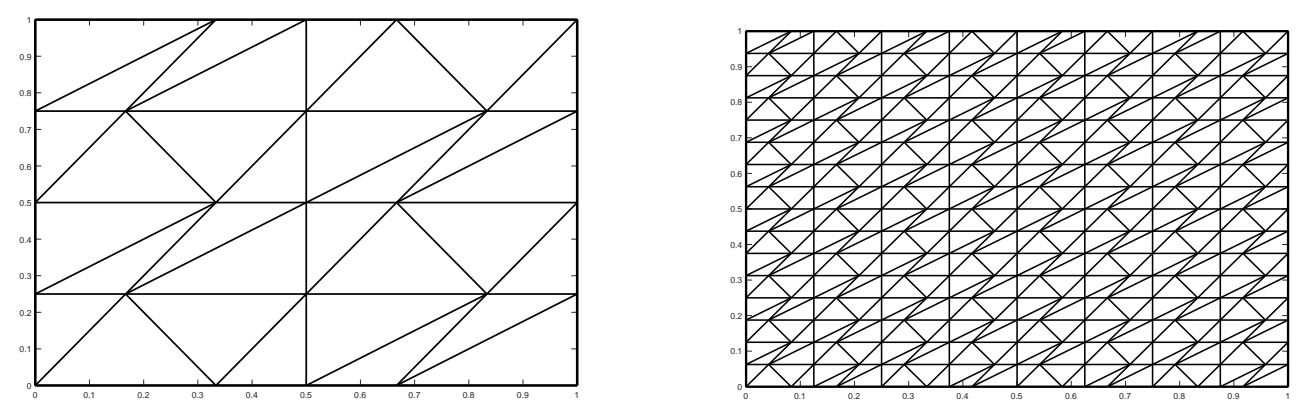

Fig. 5.2. The meshes of $\Omega=[0,1] \times[0,1]$ consisting of $O(h)$ approximate parallelograms for $n=4$ (left graph) and $n=16$ (right graph).

We plot the errors between $u_{h}$ and $u_{I}$ on $H^{1}$-seminorms for $\alpha=0,1 / 4,1 / 2$ and $\alpha=$ $1 / 3,2 / 3,2$ in Fig. 5.3. The convergence rates of $\left\|\nabla u_{h}-\nabla u_{I}\right\|_{0}$ behave much as expected. The convergence rates are almost equal to the corresponding $1+\alpha$. Such behavior shows the necessity of the condition (i) in Definition 2.1 of Condition $(\alpha, \sigma, \mu)$.
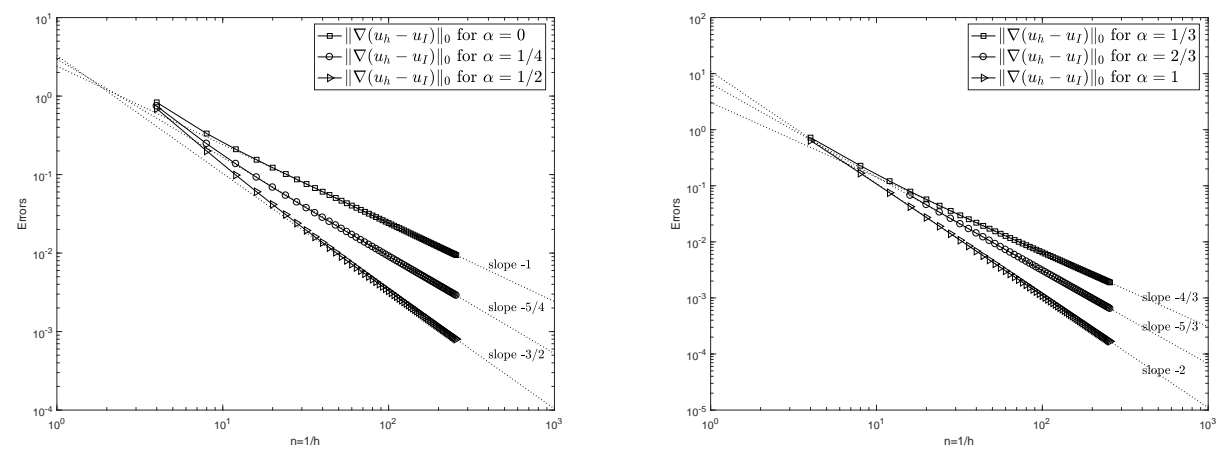

Fig. 5.3. The errors of $\left\|\nabla u_{h}-\nabla u_{I}\right\|_{0}$ for $\alpha=0,1 / 4,1 / 2$ (left graph) and $\alpha=1 / 3,2 / 3,1$ (right graph). The dotted lines indicate reference slopes.

Next, we consider the meshes containing 'bad' edges, that is $O\left(h^{1+\alpha}\right)$ approximate parallelogram property is satisfied for pairs of adjacent triangles in most parts except for a region 
of size $h^{2 \sigma}$. For given $0 \leq \sigma<1$ and non-negative integer $n$, we divide $\bar{\Omega}$ into two parts, $\Omega_{1}=[0, m h] \times[0, m h]$ and $\Omega_{2}=\Omega \backslash \Omega_{1}$, where $h=1 / n$ and the integer $m$ is chosen such that the area of $\Omega_{1}$ is about $h^{2 \sigma}$. We discrete $\Omega_{1}$ as the Criss-cross pattern uniform mesh and discrete $\Omega_{2}$ as the regular pattern uniform mesh. Fig. 5.4 plots the meshes by setting $\sigma=1 / 2$ for $n=8$ and $n=16$. Clearly $\alpha \geq 1$. It is well known that the Criss-cross pattern uniform mesh is very bad for the supercloseness property. Therefore, the convergence order $h^{2-\sigma}$ of $\left\|\nabla u_{h}-\nabla u_{I}\right\|_{0}$ can be expected. This is verified by Fig. 5.5 which plots the errors in $H^{1}$-seminorms for $\sigma=0,1 / 2,1$ and $\sigma=1 / 3,2 / 3$.
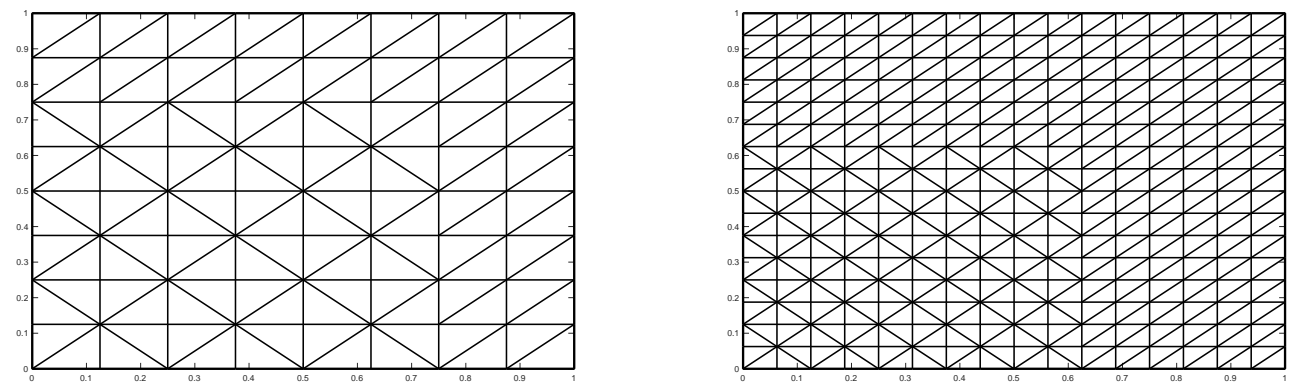

Fig. 5.4. The meshes of $\Omega=[0,1] \times[0,1]$ construted by setting $\sigma=1 / 2$ for $n=8$ (left graph) and $n=16$ (right graph).
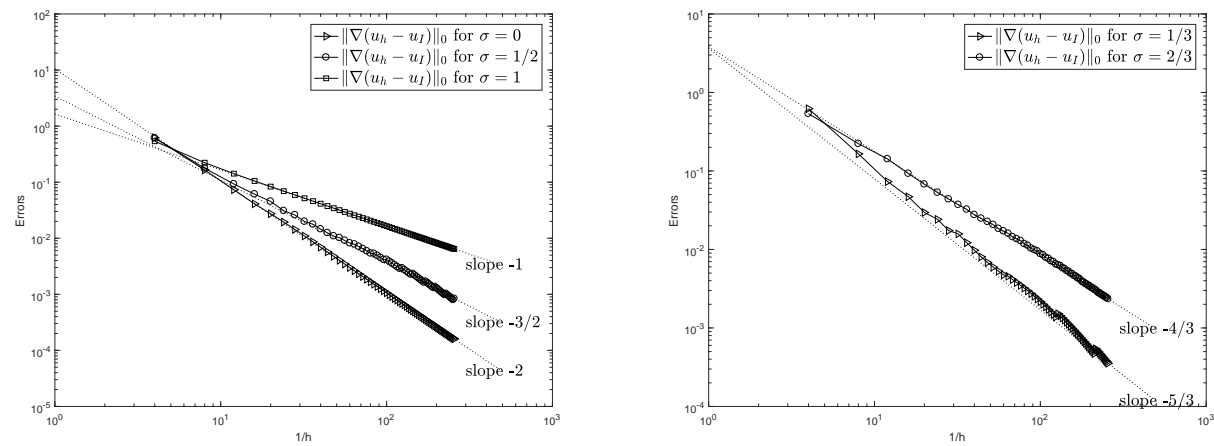

Fig. 5.5. The errors of $\left\|\nabla u_{h}-\nabla u_{I}\right\|_{0}$ for $\sigma=0,1 / 2,1$ (left graph) and $\sigma=1 / 3,2 / 3$ (right graph). The dotted lines indicate reference slopes.

Then we construct meshes by perturbing the boundary elements of the regular uniform meshes such that two boundary triangles $\tau_{e}$ and $\tau_{e^{\prime}}$ sharing a boundary node are $O(h)$ approximate congruent triangles (cf. $(2.2))$ and $\# \mathcal{N}_{2, h}^{B}=O\left(h^{-2 \mu}\right)$. Clearly, $\sigma=\mu$. Fig. 5.6 plots the perturbed mesh for $n=15$ and $\mu=1 / 4$ in the left graph and the errors of $\left\|\nabla u_{h}-\nabla u_{I}\right\|_{0}$ for $\mu=1 / 2,1 / 3,1 / 4,0$ in the right graph. The convergence order is about $O\left(h^{2-\mu}\right)$ which is better than our theoretical result in Theorem 3.1.

Then we consider the superconvergence of the numerical solution based on the Polynomial Preserving Recoverry technique. Fig. 5.7 plots the errors of $\left\|\nabla u-\nabla u_{h}\right\|_{0},\left\|\nabla u_{h}-\nabla u_{I}\right\|_{0}$ and 

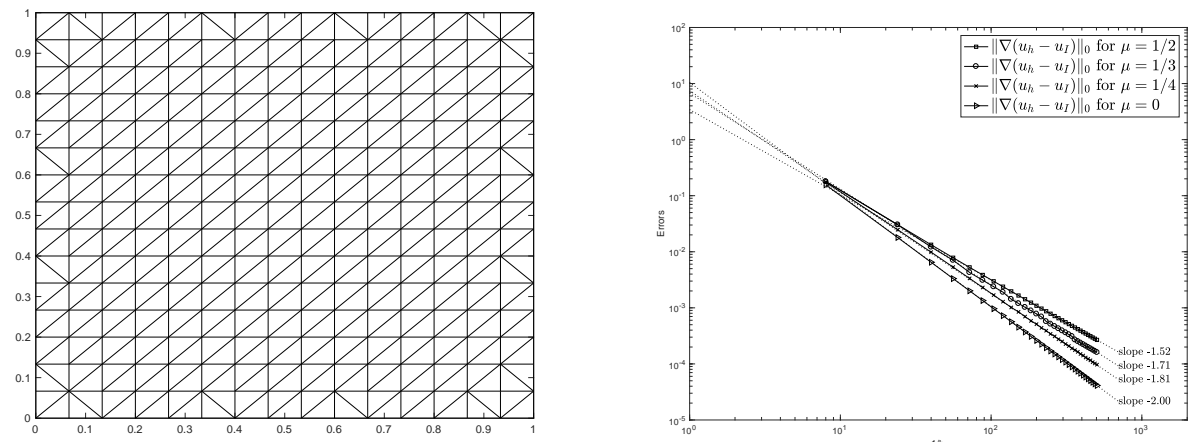

Fig. 5.6. The mesh by perturbing the regular uniform mesh for $n=15$ and $\mu=1 / 4$ (left graph) and the errors of $\left\|\nabla u_{h}-\nabla u_{I}\right\|_{0}$ for $\mu=1 / 2,1 / 3,1 / 4,0$ (right graph). The dotted lines indicate reference slopes.

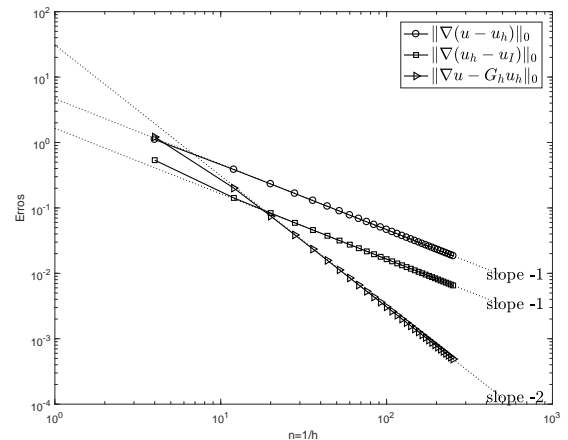

(a) Criss-Cross pattern

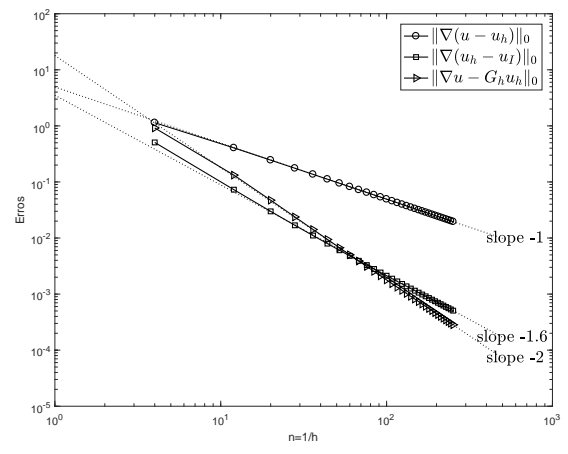

(c) Chevron pattern

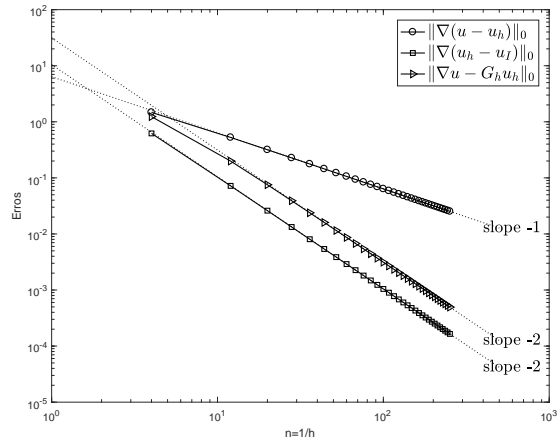

(b) regular pattern

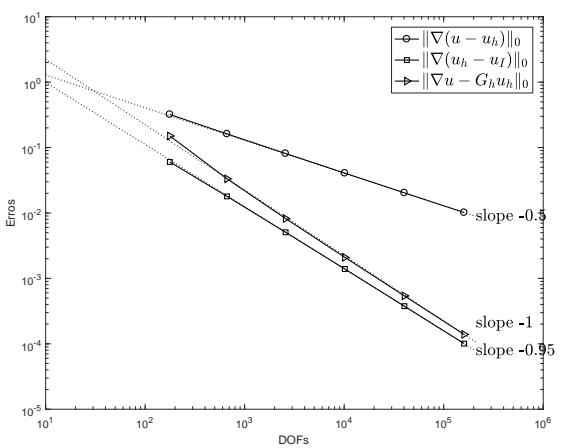

(d) Delaunay mesh

Fig. 5.7. The errors of $\left\|\nabla u-\nabla u_{h}\right\|_{0},\left\|\nabla u_{h}-\nabla u_{I}\right\|_{0}$ and $\left\|\nabla u-G_{h} u_{h}\right\|_{0}$ for Criss-cross pattern uniform mesh (a), regular pattern uniform mesh (b), Chevron pattern uniform mesh (c) and the Delaunay mesh (d). 
$\left\|\nabla u-G_{h} u_{h}\right\|_{0}$ for Criss-cross pattern uniform mesh, regular pattern uniform mesh, Chevron pattern uniform mesh and the Delaunay mesh. We see that the convergence order of $\left\|\nabla u-G_{h} u_{h}\right\|_{0}$ is almost $O\left(h^{2}\right)$ although the convergence order of $\left\|\nabla u_{h}-\nabla u_{I}\right\|_{0}$ is smaller except for the regular pattern uniform mesh, which shows that the PPR is a powerful tool to improve the gradients of the finite element solutions.

Finally, we verify the asymptotical exactness of the a posteriori error estimator $\eta_{h}$ (cf. (4.13)). Table 5.1 shows the errors of $u_{h}$ in $H^{1}$-seminorms and the a posteriori error estimators on three kinds of meshes for different mesh sizes. We watch the a posteriori error estimators converge to the corresponding $\left\|\nabla u-\nabla u_{h}\right\|_{0}$ very quickly.

Table 5.1: The errors of $\left\|\nabla u-\nabla u_{h}\right\|_{0}$ and the a posteriori error estimators on Criss-cross pattern uniform mesh, regular pattern uniform mesh, Chevron pattern uniform mesh for different mesh sizes.

\begin{tabular}{|c|c|c|c|c|c|c|}
\hline & \multicolumn{2}{|c|}{ regular pattern } & \multicolumn{2}{c|}{ Chevron pattern } & \multicolumn{2}{c|}{ Criss-cross pattern } \\
\hline $1 / h$ & $\left|u-u_{h}\right|_{1}$ & $\eta_{h}$ & $\left|u-u_{h}\right|_{1}$ & $\eta_{h}$ & $\left|u-u_{h}\right|_{1}$ & $\eta_{h}$ \\
\hline 4 & $1.47 E+00$ & $1.28 E+00$ & $1.14 E+00$ & $1.10 E+00$ & $1.10 E+00$ & $1.28 E+00$ \\
12 & $5.26 E-01$ & $5.24 E-01$ & $4.05 E-01$ & $4.06 E-01$ & $3.86 E-01$ & $4.09 E-01$ \\
20 & $3.18 E-01$ & $3.17 E-01$ & $2.45 E-01$ & $2.45 E-01$ & $2.32 E-01$ & $2.38 E-01$ \\
28 & $2.27 E-01$ & $2.27 E-01$ & $1.76 E-01$ & $1.75 E-01$ & $1.66 E-01$ & $1.68 E-01$ \\
36 & $1.77 E-01$ & $1.77 E-01$ & $1.37 E-01$ & $1.37 E-01$ & $1.29 E-01$ & $1.30 E-01$ \\
44 & $1.44 E-01$ & $1.44 E-01$ & $1.12 E-01$ & $1.12 E-01$ & $1.06 E-01$ & $1.06 E-01$ \\
52 & $1.23 E-01$ & $1.23 E-01$ & $9.47 E-02$ & $9.47 E-02$ & $8.95 E-02$ & $8.98 E-02$ \\
60 & $1.06 E-01$ & $1.06 E-01$ & $8.21 E-02$ & $8.21 E-02$ & $7.75 E-02$ & $7.77 E-02$ \\
68 & $9.37 E-02$ & $9.37 E-02$ & $7.25 E-02$ & $7.25 E-02$ & $6.84 E-02$ & $6.85 E-02$ \\
76 & $8.38 E-02$ & $8.38 E-02$ & $6.49 E-02$ & $6.48 E-02$ & $6.12 E-02$ & $6.13 E-02$ \\
84 & $7.58 E-02$ & $7.58 E-02$ & $5.87 E-02$ & $5.87 E-02$ & $5.54 E-02$ & $5.55 E-02$ \\
92 & $6.92 E-02$ & $6.92 E-02$ & $5.36 E-02$ & $5.36 E-02$ & $5.06 E-02$ & $5.06 E-02$ \\
\hline
\end{tabular}

Acknowledgments. The research of authors was supported by NSFC 11471031, 91430216, 11525103, 91630309 and 11601026; NSAF U1530401 and NSF DMS-1419040; the Hunan Provincial Natural Science Foundation of China (NO. 2019JJ50572).

\section{References}

[1] C. AB., COMSOL MultiPhysics User's Guide, 3.5a ed., 2008.

[2] M. Ainsworth And J.T. Oden, A posteriori error estimation in finite element analysis, Comput. Methods Appl. Mech. Engrg., 142 (1997), 1-88.

[3] R.E. Bank and J.C. Xu, Asymptotically exact a posteriori error estimators, Part I: Grid with superconvergence, SIAM J. Numer. Anal., 41 (2003), 2294-2312.

[4] S.C. Brenner and L.R. Scott, The mathematical theory of finite element methods, Springer, New York, third ed., 2008.

[5] C. Carstensen and S. Bartels, Each averaging technique yields reliable a posteriori error control in FEM on unstructured grids. Part i: Low order conforming, nonconforming, and mixed FEM, Math. Comp., 71 (2002), 945-969.

[6] T. Tang and J.C. Xu, Topics on adaptive finite element methods, in Adaptive Computations: Theory and Algorithms, Science Press, Beijing, 2007. 
[7] Y. Du, H.J. Wu, and Z.M. Zhang, Superconvergence analysis of linear FEM based on the polynomial preserving recovery and Richardson extrapolation for Helmholtz equation with high wave number, arXiv:1703.00156, (2017).

[8] E. Hinton and B. Irons, Least squares smoothing of experimental data using finite elements, Strain, 4 (1968), 24-27.

[9] I. Hlaváček and M. Křížek, On a superconvergent finite element scheme for elliptic systems. ii. boundary conditions of Newton's or Neumann's type, Aplikace matematiky, 32 (1987), 200-213.

[10] W. Hoffmann, A. Schatz, L. Wahlbin, and G. Wittum, Asymptotically exact a posteriori estimators for the pointwise gradient error on each element in irregular meshes. part 1: A smooth problem and globally quasi-uniform meshes, Math. Comp., 70 (2001), 897-909.

[11] B. Irons, Least square surface fitting by finite elements, and an application to stess smoothing, Aero. Stree. Meno., ASM 1524, (1967).

[12] A.M. Lakhany, I. Marek, and J. R. Whiteman, Superconvergence results on mildly structured triangulations, Comput. Methods Appl. Mech. Engrg., 189 (2000), 1-75.

[13] A. Naga and Z.M. Zhang, A posteriori error estimates based on the polynomial preserving recovery, SIAM J. Numer. Anal., 42 (2004), 1780-1800.

[14] A. Naga and Z.M. Zhang, The polynomial-preserving recovery for higher order finite element methods in 2d and 3d, Discrete Cont. Dyn.-B, 5 (2005), 759-798.

[15] J.P. Wang, Superconvergence analysis for finite element solutions by the least-squares surface fitting on irregular meshes for smooth problems, J. Math. Study, 33 (2000), 229-243.

[16] H.J. Wu and Z.M. Zhang, Can we have superconvergent gradient recovery under adaptive meshes?, SIAM J. Numer. Anal., 45 (2007), 1701-1722.

[17] J.C. Xu, An introduction to multigrid convergence theory. 1996, preprint.

[18] J.C. Xu and Z.M. Zhang, Analysis of recovery type a posteriori error estimators for mildly structured grids, Math. Comp., 73 (2003), 1139-1152.

[19] N.N. Yan and A.H. Zhou, Gradient recovery type a posteriori error estimates for finite element approximations on irregular meshes, Comput. Methods Appl. Mech. Engrg., 190 (2001), 42894299.

[20] Z.M. Zhang, Polynomial preserving gradient recovery and a posteriori estimate for bilinear element on irregular quadrilaterals, Internat. J. Numer. Anal. Model., 1 (2004), 1-24.

[21] Z.M. Zhang, Polynomial preserving recovery for anisotropic and irregular grids, J. Comput. Math., 22 (2004), 331-340.

[22] Z.M. Zhang and B. Li, Analysis of a class of superconvergence patch recovery techniques for linear and bilinear finite elements, Numer. Meth. Part. D. E., 15 (1999), 151-167.

[23] Z.M. Zhang and A. Naga, A new finite element gradient recovery method: Superconvergence property, SIAM J. Sci. Comput., 26 (2005), 1192-1213.

[24] O.C. Zienkiewicz and J.Z. Zhu, The superconvergent patch recovery and a posteriori error estimates. Part 2: Error estimates and adaptivity, Internat. J. Numer. Methods Engrg., 33 (1992), $1365-1382$.

[25] O. Zienkiewicz and J.Z. Zhu, The superconvergent patch recovery and a posteriori error estimates. Part 1: The recovery technique, Internat. J. Numer. Methods Engrg., 33 (1992), 1331-1364. 